\title{
PERFIL SOCIODEMOGRÁFICO E ADESÃO AO TRATAMENTO DE DEPENDENTES DE ÁLCOOL EM CAPS-AD DO PIAUÍ
}

\author{
Sociodemographic profile and adhesion to treatment for alcohol dependents at CAPS- \\ ad in Piauí state \\ Perfil socio-demográfico y la adhesión al tratamiento de la dependencia del alcohol en \\ CAPS-ad do Piauí
}

Claudete Ferreira de Souza Monteiro
Isadora Elisa de Moura Albuquerque

\author{
Leandro Carvalho Moura Fé2 \\ Michelly Gomes da Silva ${ }^{5}$
}

\author{
Maycon Alex Cavalcante Moreira ${ }^{3}$ \\ Mauro Cezar Passamani ${ }^{6}$
}

\section{RESUMO}

A pesquisa tem como objetivo levantar o perfil sociodemográfico e a adesão ao tratamento de dependentes do álcool no Centro de Atenção Psicossocial Álcool e Drogas. Trata-se de um estudo de natureza quantitativa, realizado no município de Teresina-PI. Os dados foram colhidos através do levantamento de informações presentes nos prontuários dos 227 sujeitos participantes desta pesquisa. Verificou-se que a maioria dos usuários era do sexo masculino $(88,9 \%)$, com idade entre 19 e 59 anos $(87,6 \%)$, solteiros (50,2\%), com ensino fundamental (40\%), empregados (48,6\%) e católicos (42,7\%). A maior parte dos sujeitos faz uso diário de bebida alcoólica $(55, \%)$ e $29 \%$ fazem uso de drogas ilícitas, sendo a maconha a mais consumida (95,3\%). Em relação à adesão, 43,1\% permaneceram em tratamento após três meses de admissão no CAPSad. Portanto, constatou-se a grande importância desse centro, com base em uma quantidade satisfatória de pacientes que aderem e que permanecem com 0 tratamento.

Palavras-chave: Enfermagem. Serviços Comunitários de Saúde Mental. Alcoolismo.

\begin{abstract}
The objective of this current study is to learn the Sociodemographic profile and adhesion to treatment for alcohol dependents at CAPS-ad. This is a quantitative study carried out at CAPS-as located in the city of Teresina, PI. The data was collected from the charts of 227 people who participated in the study. It was learned that the majority of the users were: male $(88.9 \%)$; from the age of 19 to 59 years $(87.6 \%)$; single $(50.2 \%)$, having grade school education (40\%); employed $(48.6 \%)$ and Catholic $(42.7 \%)$. Many of the subjects drank alcohol daily (55\%) and (29\%) used illegal drugs, with marijuana being consumed the most (95.3\%). In regards to adhesion, (43.1\%) remained in treatment after three months of admission to CAPS-ad. However, it was possible to verify the great importance of this center through the satisfactory quantity of patients who adhered to and remained in treatment.
\end{abstract}

Keywords: Nursing. Community Mental Health Services. Alcoholism.

\section{Resumen}

El presente estudio tiene como objetivo analizar el perfil sociodemográfico e y la adhesión al tratamiento de la dependencia del alcohol en CAPS-ad. Se trata de un estudio de naturaleza cuantitativa, realizada en el CAPS-ad localizado en el municipio de Teresina, Piauí. Los datos fueron colectados analizando informaciones presentes en los prontuarios de los 227 sujetos participantes de esta investigación. Se verificó que la mayoría de los usuarios era del sexo masculino (el 88,9\%), con edad entre 19 a 59 años (el $87.6 \%$ ) y solteros (el $50,2 \%$ ), con enseñanza fundamental (el 40\%), empleados (el 48,6\%), y católicos (el 42,7\%). La mayor parte de los sujetos hace uso diario de bebida alcohólica (el 55,\%), y el $29 \%$ hace uso de drogas ilícitas, siendo la marihuana la más consumida (el $95,3 \%$ ). En relación a la adhesión (el 43,1\%) permanecieron en tratamiento después de tres meses a partir de la admisión a CAPS-ad. Por lo tanto, se constató la gran importancia de ese centro, a través de una cantidad satisfactoria de pacientes que adhieren y que permanecen con el tratamiento

Palabras clave: Enfermería. Servicios Comunitarios de Salud Mental. Alcoholismo

'Doutora em Enfermagem. Professora Adjunto da Universidade Federal do Piauí (Graduação e Mestrado em Enfermagem) e docente da NOVAFAPI. Grupo de Estudos Sobre Enfermagem, Violência e Saúde Mental. Teresina- PI. Brasil. E-mail: claudetefmonteiro@hotmail.com, ${ }^{2}$ Enfermeiro do CAPS-ad e do PSF em Luzilândia-PI. Brasil. E-mail: leandromourafe@hotmail.com, ${ }^{3}$ Enfermeiro. Revisor de contas do CGSUS (Coordenação Geral do SUS). Especialista em Saúde da Família. Teresina-PI. Brasil. E-mail: mayconcmoreira@yahoo.com² Enfermeira. Especialista em Saúde da Família. Teresina-PI. Brasil. E-mail: isadoraealbuquerque@hotmail.com5 Enfermeira. Especialista em Saúde da Família. Teresina-PI. Brasil. E-mail: michellygs@hotmail.com²Médico. Mestre em Ciências da Saúde. Docente da Universidade Federal do Piauí. Diretor do CAPS-ad em Teresina-PI. Brasil. E-mail: mpassamani@globo.com 


\section{INTRODUÇÃO}

0 álcool é uma substância que acompanha a humanidade desde seus primórdios e sempre ocupou um local privilegiado em todas as culturas. No entanto, o conceito de alcoolismo surgiu no século XVIII, e só a partir do século XX começaram estudos sistematizados para entende-lo e considerá-lo doença, em função das consequências para os usuários e pelo impacto em termos de mortalidade e incapacidade junto às populações. ${ }^{1,2}$

Nos dias atuais, o termo alcoolismo vem sendo substituído por Síndrome de Dependência do Álcool (SDAS), a qual se caracteriza por uma dependência do álcool que ocorre aos poucos, eliminando a visão dicotômica de "tudo ou nada" implícita no termo alcoolismo ${ }^{3}$.

Em relação à etiologia do alcoolismo não existe um fator universal que a explique, pois todos que consomem bebida alcoólica apresentam possibilidade de se tornarem dependentes, sendo esta possibilidade maior ou menor conforme a interação dos fatores de vulnerabilidade (biológicos, psicológicos ou sociais). ${ }^{4}$

Entretanto, o uso indevido de álcool produz efeitos psicológicos, sociais, culturais, jurídicos, políticos e econômicos que acarretam prejuízos incalculáveis, tais como: redução das condições e qualidade de vida para o usuário e familiares, incapacidades biopsicossociais que levam o sujeito a perder oportunidades no processo produtivo, afetivo e familiar bem como surgimento de práticas de ações antissociais. ${ }^{4,5}$

A dependência de álcool no Brasil tem mostrado uma prevalência variada de $3 \%$ a $10 \%$ na população geral adulta, sendo considerada a substância psicotrópica mais consumida no país, provocando elevada busca por assistência médica dos dependentes. Segundo o Ministério da Saúde, o alcoolismo é responsável por $50 \%$ das internações psiquiátricas masculinas, $20 \%$ em unidade de gastroenterologia e $90 \%$ das internações por dependência de drogas em hospitais psiquiátricos brasileiros, sendo que pessoas com este problema são quatro vezes mais hospitalizadas que a população em geral., 2,6

0 diagnóstico de dependência de álcool é feito quando há um padrão de uso disfuncional da substância evidenciado durante um ano. É necessário três ou mais das seguintes manifestações para fechar o diagnóstico: um desejo forte ou senso de compulsão para consumir a substância; dificuldade em controlar o comportamento de consumir a substância em termos de início, término ou níveis de consumo; e estado de abstinência quando o uso da substância cessou ou foi reduzido.?

Os dependentes de álcool sob a ótica da avaliação de enfermagem geralmente revelam retardo ou hiperatividade psicomotora, alteração ou déficit de funcionamento social e familiar, aparência e hábitos higiênicos mal conservados, padrões de sono e funções psíquicas alterados, sexualidade e despertar alterados, comprometimento no padrão alimentar, dentre outros sintomas. ${ }^{5}$

0 tratamento inclui alguns serviços como avaliação diagnóstica, aconselhamentos e cuidados de saúde em geral. Além disso, há vários modelos de tratamento para os dependentes utilizados no Brasil e no mundo; dentre eles, podemos citar: hospitalar, ambulatorial e grupos de autoajuda. Independente de qual seja a escolha do indivíduo e de sua familia, a recuperação não está unicamente ligada à abstinência total, mas sim, à conquista de sua autonomia., ${ }^{8,9}$

Em virtude de os índices sobre o uso e abuso de álcool se manterem elevados, a existência de centros de atendimento compatíveis com essa realidade tornam-se necessários, tendo como objetivo promover iniciativas no âmbito comunitário bem como na assistência direta ao indivíduo e a sua família. ${ }^{9}$

Do ponto de vista do modelo de assistência psiquiátrica, a reorganização dos serviços e das ações de saúde mental fez surgir dois novos serviços de atenção representados pelos Núcleos de Atenção Psicossocial (NAPS) e pelos Centros de Atenção Psicossocial (CAPS).

No ano de 2002, o Ministério da Saúde através das Portarias n 336/GM e 816/GM regulamentou o atendimento do dependente de drogas e álcool em Centros de Atenção Psicossocial (CAPS-ad). Essa regulamentação deu início a uma mudança no cenário do atendimento a pacientes dependentes do álcool, antes atendidos principalmente em hospitais psiquiátricos. ${ }^{8} 0$ CAPS-adéuma unidade de atendimento em saúde mental, com equipe multiprofissional composta principalmente por médico, enfermeiro, psicólogo, terapeuta ocupacional e assistente social que realiza atendimentos individuais, atendimentos em grupos e visitas domiciliares. ${ }^{8}$

Neste centro a assistência de enfermagem deve incluir os familiares, tanto na participação em entrevistas individuais, como em grupos de apoio para orientação e acolhimento do cliente, pois a família pode ser importante no auxílio às mudanças de comportamento e desenvolvimento de um estilo de vida mais saudável. Além disso, não deve deixar de observar se a família tem dificuldade no manejo, pois isso pode prejudicar o decorrer do tratamento. ${ }^{9,5}$

Com base nos inúmeros casos de alcoolismo citados na literatura e confirmados na prática de enfermagem, e em virtude das consequências negativas que o uso descontrolado do álcool traz ao usuário, percebe-se a extrema relevância em conhecer o número de pacientes que aderem ao tratamento em CAPS-ad, porque essa adesão é, e certamente será, difícil, exigindo perseverança, pois deixar de consumir bebidas alcoólicas é um processo doloroso pelo qual muitos pacientes não conseguem passar. Portanto, o presente artigo buscou levantar o perfil sociodemográfico e a adesão ao tratamento por dependentes de álcool e drogas em CAPS-ad.

\section{METODOLOGIA}

0 presente estudo é de natureza quantitativa e documental, realizado no CAPS-ad na região sul do município de Teresina-PI. Este Centro funciona há seis anos e é composto por duas equipes multiprofissionais, uma em cada turno, cuja composição é feita por psiquiatra, psicólogo, enfermeiro, terapeuta ocupacional, assistente social, educador físico e auxiliar de enfermagem. 
Os dados foram levantados no período de julho a setembro de 2007, junto às informações presentes nos prontuários dos dependentes de álcool do CAPS-ad. 0 universo foi composto por 1.043 prontuários, nos quais foi obtida uma amostra do tipo probabilística sistemática (realiza-se um sorteio de um número de 1 a 5 para efeito de início de seleção). Como se trata de uma população finita, a amostra foi redimensionada para 227 prontuários e os dados coletados por meio de formulário e as informações coletadas foram processadas eletronicamente por meio do programa Statistical Product and Service Solution - SPSS.

Estes dados foram levantados buscando perfil sociodemográfico com as seguintes variáveis (sexo, idade, estado civil, escolaridade, ocupação, religião), além de dados que identificassem a característica do padrão de consumo de álcool e drogas ilícitas utilizado pelos sujeitos ao buscarem o serviço e informações que apontassem a adesão ao tratamento no CAPS-ad como tempo de permanência em tratamento e idade do dependente que aderiu ao tratamento por mais de três meses. A adesão é considerada quando o cliente permanece acima de três meses em tratamento, podendo ter, inclusive, sua alta a partir do terceiro mês a depender da gravidade da situação.

0 estudo recebeu autorização da direção do CAPS-ad e aprovação pelo Comitê de Ética em Pesquisa da Faculdade NOVAFAPI cujo número do processo é 0073.0.043.000-07.

\section{RESULTADOS}

A amostra estudada mostra predominância de dependentes do sexo masculino (88,9\%), solteiros $(50,2 \%)$, com idade entre 19 e 59 anos $(87,6 \%)$ e empregados $(48,6 \%)$. Quanto à religião, observou-se que grande parte da amostra é composta por pessoas católicas (42,7\%) e, no que diz respeito à escolaridade, a maior concentração da amostra tem apenas o ensino fundamental (40\%) (Tabela 1).

Tabela 1- Perfil sociodemográfico da população em estudo. Teresina/PI- 2007. n=227

\begin{tabular}{|c|c|c|}
\hline VARIÁVEIS & $\mathrm{N}$ & $\%$ \\
\hline \multicolumn{3}{|l|}{ SEXO } \\
\hline Masculino & 202 & 89,9 \\
\hline Feminino & 25 & 11,1 \\
\hline \multicolumn{3}{|l|}{ FAIXA ETÁRIA } \\
\hline $15-18$ & 13 & 5,7 \\
\hline $19-59$ & 199 & 87,7 \\
\hline 60 e mais & 15 & 6,6 \\
\hline \multicolumn{3}{|l|}{ ESTADO CIVIL } \\
\hline Solteiro & 114 & 50,2 \\
\hline Casado & 103 & 45,4 \\
\hline Divorciado & 06 & 2,6 \\
\hline Viúvo & 04 & 1,7 \\
\hline \multicolumn{3}{|l|}{ OCUPAÇÃO } \\
\hline empregado & 110 & 48,5 \\
\hline desempregado & 81 & 35,7 \\
\hline estudante & 28 & 12,3 \\
\hline aposentado & 8 & 3,5 \\
\hline \multicolumn{3}{|l|}{ RELIGIÃO } \\
\hline católica & 97 & 42,7 \\
\hline evangélica & 16 & 7,0 \\
\hline não referenciada & 88 & 38,8 \\
\hline outros & 26 & 11,4 \\
\hline \multicolumn{3}{|l|}{ ESCOLARIDADE } \\
\hline não alfabetizado & 16 & 7,0 \\
\hline ensino básico & 49 & 21,6 \\
\hline ensino fundamental & 91 & 40,1 \\
\hline ensino médio incompleto & 26 & 11,5 \\
\hline ensino médio completo & 36 & 5,7 \\
\hline ensino superior incompleto & 06 & 2,6 \\
\hline ensino superior completo & 03 & 1,3 \\
\hline
\end{tabular}


Ao se relacionar o tempo de uso de bebida alcoólica e a faixa etária, obteve-se uma média de 3 anos $(\mathrm{dp}=1,5)$ na faixa etária compreendida entre 15 e 18 anos e de 22,3 anos $(d p=10,9)$ entre 19 e 59 anos de idade. Para a faixa etária de 60 anos ou mais, a média apresentada foi de 40,9 anos $(\mathrm{dp}=13,1)$ (Tabela 2).

Tabela 2- Média e desvio-padrão do tempo de uso de bebida alcoólica segundo faixas etárias. Teresina/Pl- 2007

\begin{tabular}{lll}
\hline Faixa etária (anos) & Média & Desvio-Padrão \\
\hline $15-18$ & 3,0 & 1,5 \\
$19-59$ & 22,3 & 10,9 \\
60 e mais & 40,9 & 13,1 \\
\hline
\end{tabular}

Os dados registrados nos prontuários mostram que 0 uso de bebida alcoólica pelos sujeitos pesquisados ao darem entrada nos CAPSad estava presente como uso diário em $55 \%$ da amostra, seguido de 35,2\% para mais de três vezes por semana e $8,8 \%$ para uma a duas vezes por semana e dois registro de uso quinzenal (Tabela 3).

Tabela 3 - Frequência do uso da bebida alcoólica na população do estudo. Teresina/PI - 2007

\begin{tabular}{lll}
\hline & $\mathrm{N}$ & $\%$ \\
\hline Diariamente & 125 & 55 \\
Mais de três vezes por semana & 80 & 35,2 \\
Uma a duas vezes por semana & 20 & 8,8 \\
Quinzenalmente & 2 & 0,8 \\
\hline
\end{tabular}

No que se refere ao uso de drogas ilííitas pela população em estudo, verifica-se que a maioria da amostra $(71 \%)$ referiu não usar. Entre aqueles que utilizam drogas ilícitas, a maconha foi a mais frequentemente referida (95,3\%).

Em relação à média de permanência em tratamento no CAPS-ad, verificou-se que, dos 227 dependentes de álcool e outras drogas que compõem a amostra, houve interrupção de 129 (56,8\%) e permanência de $98(43,1 \%)$ com mais de três meses de tratamento. Para os $43,1 \%$ que deram continuidade, foi possível observar quanto tempo permaneceram em tratamento levando-se em conta a faixa etária. Assim, na faixa etária entre 15 e 18 anos, a média encontrada foi de 5,5 meses ( $d p=4,3$ ); para a faixa compreendida entre 19 e 59 anos de idade, foi de 6,3 meses $(d p=5,1)$ e para 60 anos e mais, a média em meses foi de 9,8 e desvio-padrão de 10,4 (Tabela 4). Ao levantar dados referentes à procura por tratamento, observou-se que $\mathrm{a}$ maioria dos sujeitos $(50,6 \%)$ não o fez por iniciativa própria e que 49,3 procuraram o tratamento por iniciativa própria.

Tabela 4 - Media e desvio-padrão do tempo de permanência em CAPS-ad (meses) por faixa etária, no grupo composto de $43,1 \%$ da amostra. Teresina/PI - 2007.

\begin{tabular}{lll}
\hline Faixa etária & $\begin{array}{l}\text { Média de Permanência } \\
\text { (meses) }\end{array}$ & Desvio-padrão \\
\hline $15-18$ & 5,5 & 4,3 \\
$19-59$ & 6,3 & 5,1 \\
60 e mais & 9,8 & 10,4 \\
\hline Total & 6,4 & 5,6 \\
\hline
\end{tabular}

\section{DISCUSSÃO}

A análise do perfil sociodemográfico da população estudada mostra maior número do sexo masculino, o que corrobora com estudo que evidenciou questão de gênero para a busca do tratamento, indicando que as mulheres sentem vergonha da doença, resultando em afastamento do tratamento. ${ }^{10}$ No entanto, em um estudo realizado por César, em 2006, revelou uma maior adesão do sexo feminino ao tratamento, principalmente quando os grupos em tratamento eram compostos apenas por mulheres. ${ }^{11} \mathrm{Na}$ instituição pesquisada neste estudo, não há a separação por sexo dos pacientes em tratamento.

Já em relação à frequência predominante de homens, os dados encontram-se similaridades também com uma pesquisa realizada na cidade de Pelotas no Rio Grande do Sul, que mostrou 
maior prevalência do uso abusivo de álcool pelo sexo masculino $(29,2 \%)$ em relação ao sexo feminino (3,7\%), fato também constatado por estudo realizado em outro município do Rio Grande do Sul, no qual se destaca o homem fumante, de baixo nível socioeconômico mais vulnerável ao abuso e à dependência de álcool. ${ }^{12,13}$

Na análise acerca da idade dos dependentes de álcool, verificou-se que a faixa etária entre 19 e 59 anos é aquela mais assídua ao CAPS-ad, sendo estes em sua maioria solteiros, empregados e com ensino fundamental.

Os resultados revelam que, em relação à religião, os evangélicos constituem o grupo menos predominante para tratamentos em CAPS-ad. Corroborando estes dados, pesquisas mostram que os evangélicos fazem menos uso de álcool em relação aos católicos e que a prevalência do uso de álcool é maior em quem não tem religião ou não é da religião evangélica. ${ }^{14}$

Ressalta-se nos resultados que, com o aumento da idade, aumenta o tempo de uso, assim os idosos são os que apresentam a maior média de tempo de uso de bebida alcoólica. 0 alcoolismo no idoso pode estar associado a complicações de saúde, dificuldades financeiras, tabagismo e maior frequência de eventos estressores na vida. Problemas relacionados ao uso de álcool podem estar presentes em aproximadamente $1 \%$ a $10 \%$ dos idosos vivendo na comunidade. ${ }^{15}$.

Nesta pesquisa verificou-se que estes sujeitos iniciam precocemente o consumo e apresentam anos de uso desta substancia, que com o passar do tempo agrava o prognóstico, pois há um aumento da dependência, comprometendo cada vez mais as atividades do dia-a-dia e das funções biopsicossociais.

Alguns autores apontam a bebida alcoólica como uma substância psicotrópica bastante conhecida pelos adolescentes, com uma idade média de início para o uso de pouco mais de 12 anos de idade, ou seja, bem antes da idade legal para consumo. ${ }^{16}$

De acordo com os dados desta pesquisa, a droga ilícita mais utilizada pela amostra é a maconha. Uma pesquisa realizada em uma universidade de São Paulo mostrou alta prevalência do uso de drogas ilícitas em adolescentes que fazem uso de álcool; destas, a que mais se destaca também é a maconha. ${ }^{14}$

Em relação à aderência ou adesão ao tratamento, esta significa o grau em que o paciente segue as recomendações médicas, ou de outro profissional da saúde consultado, e é entendida como o retorno e a manutenção do tratamento indicado. Pode ser também chamada de obediência e permanência ao tratamento e inclui a frequência até o momento de sua alta. ${ }^{17}$

Na instituição pesquisada, o tempo pré-estabelecido para se considerar adesão, vai a partir do terceiro mês do tratamento, considerando boa adesão se o paciente tem permanência ao tratamento por um período mínimo de seis meses e muito boa quando o paciente permanece por mais de seis meses. Foi possível identificar que a maioria da amostra pesquisada não dá continuidade ao tratamento. Estes dados corroboram estudo realizado no município do Rio Grande do Sul, cujos resultados demonstram que $53,45 \%$ dos usuários não permanecem em tratamento na instituição, apesar de haver grande procura pelo serviço. ${ }^{18}$

A continuidade ao tratamento para dependentes de álcool pode ser considerada um percurso difícil de atingir, pois muitos o iniciam, e à medida que obstáculos como recaídas e as dificuldades em abandonar a bebida falam mais alto que 0 desejo de não beber, poucos conseguem permanecer em tratamento sem fazer uso da bebida alcoólica. Para vencer estes obstáculos é necessário antes de tudo que o dependente se conscientize de sua doença e dos males que esta causa, como dificuldades na vida profissional, nos relacionamentos e no convívio familiar.

Para os dependentes de álcool e outras drogas que deram continuidade, houve uma permanência em tratamento no CAPS-ad superior a seis meses, principalmente na faixa etária compreendida entre 19 a 59 anos, na qual também se concentra o maior número de sujeitos. Para a faixa de 60 anos e mais, a permanência chega em torno de nove meses, mas com número menor de pessoas. Já para a faixa abaixo de 18 anos, essa permanência tem média abaixo de seis meses, indicando que há necessidade de mudança de estratégia para conseguir melhorar a permanência desse grupo ao tratamento.

0 estudo ainda aponta diferença pouco significativa entre a procura por iniciativa própria ou não entre os dependentes. Entende-se que a falta de iniciativa é uma limitação que impede a continuidade no tratamento; no entanto, se o paciente estiver consciente do seu problema e com vontade de enfrentá-lo, maior será a resolutibilidade de sua doença.

\section{CONCLUSÕES}

Neste estudo, para a amostra pesquisada, conclui-se que a adesão ainda não foi alcançada em sua maioria e, entre aqueles que continuaram o tratamento, a adesão foi considerada muito boa, permanecendo em tratamento por um período superior a seis meses. Muito embora o estudo não permitisse avaliar se durante essa permanência houve recaídas e nem quantas vezes ocorreu, o fato de permanecerem em tratamento não os exime da utilização da bebida alcoólica, mas espera-se que o tratamento seja capaz de modificar essa situação.

Como o CAPS-ad trabalha com o retorno do usuário de forma diária, semanal ou bimestralmente, essa metodologia, ao mesmo tempo em que proporciona o tratamento sem isolar o paciente do contexto social, não o impede de estar livre para o consumo do álcool, razão pela qual se deve reforçar a motivação para o tratamento, o que ressalta a importância da utilização de estratégias de prevenção de recaídas o mais precocemente possível e garantir maior adesão ao tratamento por um número cada vez maior de pessoas que procuram os CAPS-ad. 
Por se enfocar a adesão, o estudo não levantou dados dos sujeitos que não aderiam ou que abandonaram o tratamento antes de completar três meses, mas sugere-se que dados do abandono sejam levantados em estudos posteriores relacionando ao perfil desses dependentes de álcool e drogas que desistem do tratamento.

\section{REFERÊNCIAS}

1 Gigliotti A, Bessa MA. Síndrome de dependência do álcool: critérios diagnósticos. Rev Bras Psiquiatr. [on-line] 2004 maio [citado 2007 mar 19];26 (1). Disponível e: http://uww.scielo.br/pdf/rbp/v26s1/a04v26s1.pdf

2 Luis MAV, Lunetta ACF. Álcool e outras drogas: levantamento preliminar sobre a pesquisa produzida no Brasil pela enfermagem. [on-line] Rev Latino-am Enfermagem. [on-line]. 2005 nov/ dez [citado 2007 mar 19] ; 13(2). Disponível em: http://www.scielo.br/pdf/rlae/v13nspe2/ v13nspe2a18.pdf

3 Filizola CLA, Perón CJ, Nascimento MMA, Pavarini SCl, Petrilli Filho JF. Compreendendo o alcoolismo na família. Esc Anna Nery. [on-line] $2006 \mathrm{dez}$ [citado 2007 set 13] ;10 (4): 660-70. Disponível em: http:// www.portalbvsenf.eerp.usp.br/pdf/ean/v10n4/v10n4a07.pdf

4 Aliane PP, Lourenco LM, Ronzani TM. Estudo comparativo das habilidades sociais de dependentes e não dependentes de álcool. [on-line] Psicol Estud. [2006 jan/abr [citado 2007 mar 21]; 11(1). Disponível em: http:// www.scielo.br/pdf/pe/v11n1/v11n1a10.pdf

5 Miranda FAN, Simpson CA, Azevedo DM, Costa SS. O impacto negativo dos transtornos do uso e abuso do álcool na convivência familiar. Rev Eletr Enferm. [on-line] 2006 [citado 2007 abr 21] ; 8(2). Disponível em: http:/ /www.fen.ufg.br/revista/revista8_2/v8n2a07.htm

6 Laurenti R, Jorge MHPM, Gotlieb SLD. Perfil epidemiológico da morbimortalidade masculina. Cienc. Saude Colet [on- line] 2005[citado 2007 abr 10]; 10(1). Disponível em: http://www.scielo.br/pdf/csc/v10n1/ a04v10n1.pdf

7. Organização Mundial de Saúde-OMS. Classificação Estatística Internacional de Doenças e Problemas Relacionados a Saúde -CID-10. 10ª revisão, v.1. São Paulo (SP): Ed USP; 2006.

8 Ministério da Saúde (BR). Secretaria de Atenção à Saúde. Departamento de Ações Programáticas Estratégicas. Saúde mental no SUS: os Centros de Atenção Psicossocial. Brasília(DF; 2004.

9 Pillon SC, Luis MAV. Modelos explicativos para o uso de álcool e drogas e a prática da enfermagem. Rev Latino-am Enfermagem. [on-line] 2004 jul/ ago[citado 2007 abr 17]; 12(4). Disponível em: http://www.scielo.br/pdf/ rlae/v12n4/v12n4a14.pdf

10 Rossetti ACK, Santos VEP. Dependente químico: adesão ao tratamento. Rev Tec-cientEnferm. [on- line] 2006 [citado 2007 abr 20]; 4(14). Disponível em: http://www.scielo.br

11 Cesar BAL. Alcoolismo feminino: um estudo de suas peculiaridades. J Bras Psiquiatr [on-line] 2006 out [citado 2007 abr 20]; 55(3). Disponível em: http://www.scielo.br/pdf/jbpsiq/v55n3/v55n3a06.pdf
12 Costa JSD, Silveira MF, Gazalle FK, Oliveira SS, Hallaal PC, Menezes AMB, et al . Consumo abusivo de álcool e fatores associados: estudo de base populacional. Rev Saude Publica. [on-line] 2004 [citado 2007 out 5]; 38(2). Disponível em: http://www.scielo.br/pdf/rsp/v38n2/ 19790.pdf

13 Primo NLNP, Stein AT. Prevalência do abuso e da dependência de álcool em Rio Grande (RS): um estudo transversal de base populacional. Rev Psiquiatr. Rio Gd. Sul. [on-line] 2004 [citado 2007 out 05], 26(3). Disponível em: http://www.scielo.br/pdf/rprs/v26n3/ v26n3a05.pdf

14 Silva LVER, Malbergier A, Stempliuk VA, Andrade, AG. Fatores associados ao consumo de álcool e drogas entre estudantes universitários. Rev Saude Publica. [on-line] 2006 [citado 2007 out 5]; 40(2). Disponível em: http://www.scielosp.org/pdf/rsp/v40n2/ 28533.pdf

15 Almeida OP. Idosos atendidos em serviço de emergência de saúde mental: características demográficas e clínicas. Rev Bras Psiquiatr. [online] 1999 [citado 2007 out 5]; 21(1). Disponível em: http:// www.scielo.br/pdf/rbp/v21n1/v21n1a05.pdf

16 Pinsky I, Pavarino FRV. A apologia do consumo de bebidas alcoólicas e da velocidade no trânsito no Brasil: considerações sobre a propaganda de dois problemas de saúde pública. Rev Psiquiatr. Rio Gd. Sul. [on-line] 2007 [citado 2007 out 7]; 29(1). Disponível em: http:// www.revistapsiqrs.org.br/administracao/arquivos/v29n1a19.pdf

17 Kaplan HI, Sadock BJ, Grebb JA. Compêndio de psiquiatria: ciências do comportamento e psiquiatria clínica. $7^{\mathrm{a}}$ ed. Porto Alegre(RS): Artmed; 1997.

18 Pelisoli CL, Moreira AK. Caracterização epidemiológica dos usuários do Centro de Atenção Psicossocial. Rev Psiquiatr Rio Gd. Sul. [on-line] 2005 [citado 13 set 2007]; 27(3). Disponível em: http://www.scielo.br/ pdf/rprs/v27n3/v27n3a06.pdf
Recebido em 15/09/2009 Reapresentado em 04/04/2010 Aprovado em 08/09/2010 